\title{
Osteopetrosis in a six-month-old infant
}

\author{
Archana M. Agarwal ${ }^{1,2} \cdot$ Timothy M. Hanley ${ }^{1,2}$ (D) \\ Received: 26 April 2020 / Accepted: 13 May 2020 / Published online: 27 May 2020 \\ (C) Springer-Verlag GmbH Germany, part of Springer Nature 2020
}

\begin{abstract}
Osteopetrosis is a severe and frequently fatal condition characterized by the formation of thick, dense bone, with a concomitant loss of bone marrow spaces secondary to defective osteoclast function or development. Clinical symptoms include pancytopenia, hepatosplenomegaly, fractures, cranial nerve impingement, and increased cranial pressure, among others. The condition requires rapid diagnosis and clinical intervention, as hematopoietic stem cell transplantation performed early in life can be curative in the majority of cases and can prevent the irreversible neurological impairment associated with the disease. We report a six-month-old male with osteopetrosis where bone marrow examination and molecular studies allowed a definitive diagnosis. Microscopic examination of a bone marrow biopsy demonstrated increased bone formation at various stages of ossification, decreased marrow spaces, and increased osteoclasts rimming the bone. Next-generation sequencing (NGS) demonstrated that the patient was homozygous for mutations in the TCIRG1 gene, which encodes an osteoclast vacuolar proton pump and is the most common mutation associated with the autosomal recessive or infantile malignant form of osteopetrosis. The patient underwent a cord blood stem cell transplant, but unfortunately expired four months after diagnosis. This case highlights the importance of bone marrow biopsy evaluation as part of the integrated approach to diagnosing osteopetrosis.
\end{abstract}

Keywords Osteopetrosis · Osteoclast · Bone marrow biopsy · TCIRG1

\section{Introduction}

Osteopetrosis, also known as "marble bone disease" or Albers-Schonberg disease, is a rare genetic disorder characterized by defective osteoclast function or development and impaired bone resorption that results in generalized sclerosis of bones with a reduction of marrow spaces. Osteopetrosis is categorized into three types based on the pattern of inheritance: (1) an autosomal recessive (infantile malignant) form; (2) an autosomal dominant (adult) form; and (3) an X-linked form [1]. The autosomal recessive form of osteopetrosis can be further subdivided into osteoclast-rich and osteoclast-poor forms based on the number of osteoclasts present. These forms correspond to defects in osteoclast function (osteoclast-rich) or osteoclast development (osteoclast-poor) and are associated with distinct mutations [2]. The autosomal

Timothy M. Hanley

timothy.hanley@aruplab.com

1 Department of Pathology, University of Utah, Salt Lake City, UT, USA

2 ARUP Laboratories, Inc., Salt Lake City, UT, USA recessive form of osteopetrosis is associated with early onset and a poor prognosis, whereas the autosomal dominant form manifests in later childhood and is typically discovered as an incidental radiographic finding. While autosomal recessive osteopetrosis is very rare with an incidence of approximately $1: 200,000-1: 300,000$, autosomal dominant osteopetrosis is more common with an incidence of 1:20,000 [3]. There are various tests that can be used to diagnose osteopetrosis including radiographic studies, bone marrow biopsy, and molecular studies.

\section{Materials and methods}

\section{Specimen collection and processing}

Peripheral blood and bone marrow were submitted and processed in the Department of Pathology, University of Utah, and ARUP Laboratories according to established protocols. For the bone marrow specimen, an aspirate, clot section, and trephine biopsy were prepared. The aspirates were distributed into the appropriate anticoagulant (EDTA- or sodium heparin-containing tubes) for ancillary studies (flow 
cytometry, cytogenetics, molecular), and the bone marrow core biopsy and aspirate clot were submitted in containers containing 10\% neutral-buffered formalin. The clot section and biopsy were processed and stained with hematoxylin and eosin or immunohistochemical stains, as required.

\section{Immunophenotyping}

The bone marrow aspirate specimen for flow cytometry was collected in sodium heparin tubes and analyzed using a multiparameter, 10-color flow cytometer. The antibody panel included CD2, CD3, CD4, CD5, CD7, CD8, CD10, CD11b, CD13, CD14, CD16, CD19, CD20, sCD22, CD33, CD34, CD38, CD45, CD56, CD117, HLA-DR, kappa light chain, and lambda light chain.

\section{Molecular and cytogenetic analyses}

Next-generation sequencing (NGS) of over 4500 genes was performed on EDTA-anticoagulated peripheral blood from the patient and his parents. DNA was extracted using a Chemagic kit (Perkin Elmer), quantified using a Qubit (Thermo Fischer Scientific), subjected to library preparation, and sequenced on an Illumina HiSeq 4000, followed by interpretation by a pathologist. Sanger sequencing was performed to confirm reported variant(s).

Preparation of metaphase chromosomes for conventional cytogenetic analysis was performed according to established methods and analyzed using the MetaSystems Ikaros karyotyping platform.

\section{Clinical history and results}

A six-month-old male, born at term to consanguineous parents presented with increased fussiness, ocular deviation, abnormal movements, and persistent bulging of the anterior fontanelle. An MRI of the head demonstrated a Chiari malformation with supratentorial ventriculomegaly. Physical examination revealed hepatosplenomegaly. Laboratory studies demonstrated an elevated LDH of 862 units/L (normal range 163-452 units/ L) and an elevated alkaline phosphatase of 1203 units/L (normal range 134-518 units/L). A complete blood count performed at that time demonstrated pancytopenia with a marked normocytic normochromic anemia (hemoglobin of $5.2 \mathrm{~g} / \mathrm{dL}$, normal range 10.5-13.5 g/dL), a mild leukopenia (WBC of $5.2 \mathrm{k} / \mathrm{uL}$; normal range $5.5-17 \mathrm{k} / \mathrm{uL}$ ) with an absolute neutropenia (ANC of $1.3 \mathrm{k} / \mathrm{uL}$ ) and a granulocytic left shift, and a moderate thrombocytopenia $(54 \mathrm{k} / \mathrm{uL}$, normal range 150 $400 \mathrm{k} / \mathrm{uL}$ ). The WBC differential leukocyte count demonstrated a granulocytic left shift with $18 \%$ segmented neutrophils, $7 \%$ bands, $51 \%$ lymphocytes, $15 \%$ monocytes, $1 \%$ basophils, $2 \%$ eosinophils, $2 \%$ metamyelocytes, and $4 \%$ myelocytes.
Frequent (15/100 WBCs) nucleated red blood cells (RBCs) were present. Peripheral blood smear evaluation (Fig. 1) demonstrated leukoerythroblastic changes including left-shifted myeloid cells with very rare circulating blasts and nucleated RBCs. In addition, occasional dacrocytes (tear drop cells) and schistocytes were present. There was no evidence of dysplasia.

A subsequent bone marrow biopsy performed to evaluate pancytopenia demonstrated a markedly hypocellular marrow (5\%) with decreased trilineage hematopoiesis. There were markedly decreased bone marrow spaces resulting from bone overgrowth at different stages of ossification (Fig. 2). In addition, there were increased numbers of osteoblasts and osteoclasts surrounding the dense bone formation. There were no increased blasts or dysplastic changes identified. Overall, the morphologic findings were most consistent with osteopetrosis. Flow cytometric evaluation of the bone marrow aspirate demonstrated no evidence of a hematologic malignancy and chromosome analysis was normal. NGS sequencing confirmed that the patient was homozygous for a frameshift mutation (c.971dupG; p.Cys324fs) in the TCIRG1 gene, which is associated with autosomal recessive osteopetrosis [4]. NGS sequencing of the parents showed that both the mother and father were heterozygous for this mutation. A skeletal survey demonstrated findings consistent with a skeletal dysplasia characterized by markedly increased density of the bones with a halo of poor mineralization surrounding the bones, most prominent at the metaphyses. The patient was treated with packed $\mathrm{RBC}$ and platelet transfusions to bridge to transplant, and eventually underwent an umbilical cord blood stem cell transplant. Unfortunately, the patient expired of respiratory failure approximately four months after diagnosis.

\section{Discussion}

Bone marrow core biopsy evaluation is an essential part of the diagnostic work-up for osteopetrosis because of the associated pancytopenia. Due to the rare nature of the disorder, there have been no large studies evaluating the morphology of bone marrow core biopsies in osteopetrosis patients; however, several smaller case series and case reports of osteopetrosis describe bone marrow biopsies that typically demonstrate increased bone density with new woven bone formation at varying stages of ossification, as well as significant narrowing of bone marrow spaces with both marked hypocellularity and suppressed trilineage hematopoiesis [5-12]. In addition, there is abnormal bone formation with thickened, disorganized bony trabeculae [13-15]. Areas of patchy reticulin fibrosis can also be seen [16]. The characteristically abnormal bone formation seen in osteopetrosis leads to decreased marrow spaces and interferes with normal medullary hematopoiesis. 

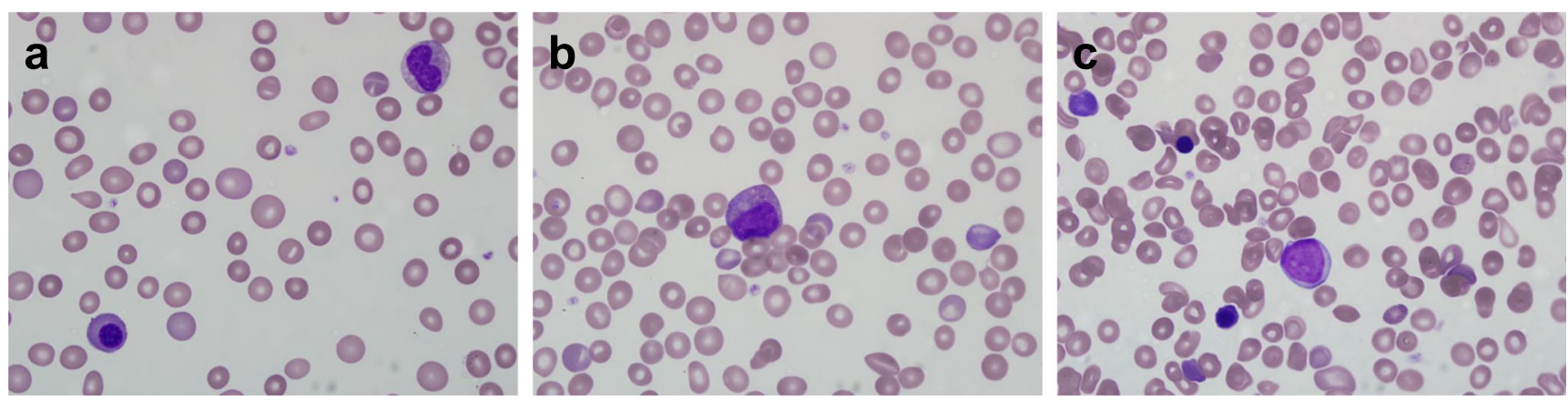

Fig. 1 Peripheral blood smear. a, b Leukoerythroblastic changes including granulocytic left shift and circulating nucleated RBCs. c Rare circulating blasts

This manifests as cytopenias, which can be severe, and increased susceptibility to infections [17]. The loss of normal medullary hematopoiesis also leads to extramedullary hematopoiesis, resulting in a leukoerythroblastic blood smear and hepatosplenomegaly. Cytopenias, leukoerythroblastic peripheral blood smears, and extramedullary hematopoiesis are consistently present in patients with autosomal recessive osteopetrosis.

The characteristic bone findings in osteopetrosis may result from mutations in a number of genes important for osteoclast function and development, including TCIRG1, CLCN7, OSTM1, SNX10, PLEKHM1, TNFSF11, TNFRSF11A, and SLC29A3. This case of osteopetrosis demonstrated a pathogenic frameshift mutation in the TCIRG1 gene, which is the most commonly mutated gene in autosomal recessive, osteoclast-rich osteopetrosis and encodes a subunit of the osteoclast vacuolar proton pump. Other common mutations in autosomal recessive, osteoclast-rich forms of osteopetrosis involve genes encoding a chloride channel protein $(C L C N 7)$ and a transmembrane protein (OSTM1) that stabilizes the chloride channel protein. All of these mutations interfere with the acidification of resorption lacunae within bone, which are necessary for the dissolution of mineralized bone matrix during bone resorption [18]. Autosomal recessive, osteoclast-poor forms of osteopetrosis which demonstrate a complete or near-complete absence of osteoclasts in bone marrow biopsies are most often associated with mutations in the genes TNFSF 11 and TNFSF11A, which encode the proteins Receptor Activator of Nuclear Kappa B Ligand (RANKL) and RANK, respectively; two proteins essential for the normal development of osteoclasts.

Treatments for osteopetrosis involve either stimulation of osteoclast function or osteoclast replacement through hematopoietic stem cell transplant. Calcitriol (1,25-dihydroxy vitamin D3) is one therapeutic agent that can be used to stimulate osteoclasts to resorb bone; however, administration typically produces only modest clinical improvement. Recombinant interferon-gamma is an additional medical treatment that improves white blood cell function and decreases trabecular bone volume. The definitive treatment is
Fig. 2 Bone marrow core biopsy. a, b Disorganized bony trabeculae, markedly decreased marrow spaces, and decreased trilineage hematopoiesis. c Numerous osteoclasts rimming trabecular bone. d CD68 immunohistochemical stain highlighting osteoclasts
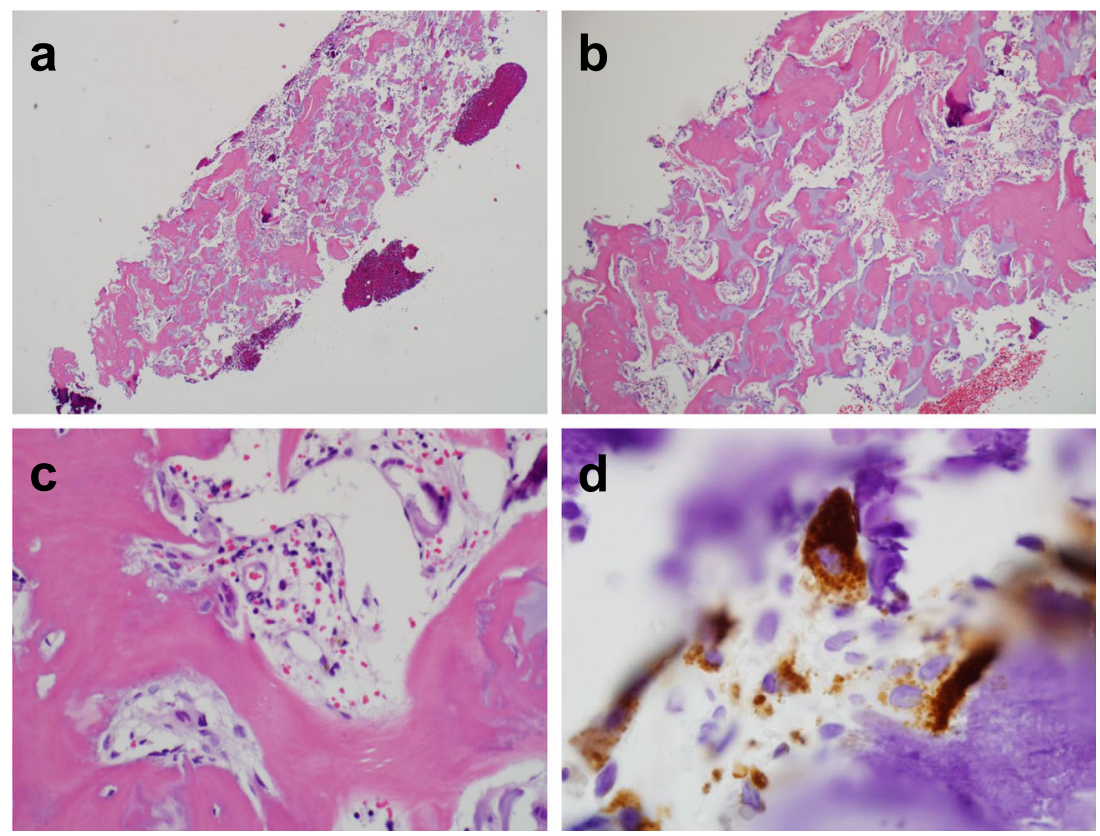
hematopoietic stem cell transplant, which is curative in patients with defects of osteoclast function or development with five-year disease-free survival rates of approximately $80-90 \%$ depending upon the donor type [19].

Although a rare disease, osteopetrosis should be a consideration in infants who present with a leukoerythroblastic peripheral blood smear, cytopenias, and hepatosplenomegaly. Despite conflicting recommendations [19, 20], this case highlights the importance of bone marrow biopsy evaluation as part of an integrated approach to diagnosing osteopetrosis.

\section{Compliance with ethical standards}

Conflict of interest The authors declare that they have no conflicts of interest.

\section{References}

1. Palagano E, Menale C, Sobacchi C, Villa A (2018) Genetics of osteopetrosis. Curr Osteoporos Rep 16(1):13-25. https://doi.org/ 10.1007/s1 1914-018-0415-2

2. Penna S, Capo V, Palagano E, Sobacchi C, Villa A (2019) One disease, many genes: implications for the treatment of osteopetroses. Front Endocrinol (Lausanne) 10:85. https://doi.org/ 10.3389 /fendo.2019.00085

3. Sobacchi C, Schulz A, Coxon FP, Villa A, Helfrich MH (2013) Osteopetrosis: genetics, treatment and new insights into osteoclast function. Nat Rev Endocrinol 9(9):522-536. https://doi.org/10. 1038/nrendo.2013.137

4. Sobacchi C, Frattini A, Orchard P, Porras O, Tezcan I, Andolina M, Babul-Hirji R, Baric I, Canham N, Chitayat D, Dupuis-Girod S, Ellis I, Etzioni A, Fasth A, Fisher A, Gerritsen B, Gulino V, Horwitz E, Klamroth V, Lanino E, Mirolo M, Musio A, Matthijs G, Nonomaya S, Notarangelo LD, Ochs HD, Superti Furga A, Valiaho J, van Hove JL, Vihinen M, Vujic D, Vezzoni P, Villa A (2001) The mutational spectrum of human malignant autosomal recessive osteopetrosis. Hum Mol Genet 10(17):1767-1773. https://doi.org/10.1093/hmg/10.17.1767

5. Barnea Slonim L, Glasser C (2018) Infantile osteopetrosis. J Pediatr Hematol Oncol 40(3):225-226. https://doi.org/10.1097/MPH. 0000000000001108

6. Diniz G, Olukman O, Calkavur S, Buyukinan M, Altay C (2015) A histologically diagnosed case with infantile osteopetrosis complicated by hypopituitarism. Case Rep Pathol 2015:786836-786835. https://doi.org/10.1155/2015/786836

7. Jain M, Mittal P, Shukla A, Kumar A (2017) Osteopetrosis in twin infants mimicking leukemia. Rev Bras Hematol Hemoter 39(4): 372-374. https://doi.org/10.1016/j.bjhh.2017.06.002
8. Everts V, de Vries TJ, Helfrich MH (2009) Osteoclast heterogeneity: lessons from osteopetrosis and inflammatory conditions. Biochim Biophys Acta 1792(8):757-765. https://doi.org/10.1016/ j.bbadis.2009.05.004

9. Helfrich MH, Aronson DC, Everts V, Mieremet RH, Gerritsen EJ, Eckhardt PG, Groot CG, Scherft JP (1991) Morphologic features of bone in human osteopetrosis. Bone 12(6):411-419. https://doi.org/ 10.1016/8756-3282(91)90030-m

10. Mazzolari E, Forino C, Razza A, Porta F, Villa A, Notarangelo LD (2009) A single-center experience in 20 patients with infantile malignant osteopetrosis. Am J Hematol 84(8):473-479. https://doi. org/10.1002/ajh.21447

11. Milgram JW, Jasty M (1982) Osteopetrosis. A morphological study of twenty-one cases. J Bone Joint Surg Am 64(6):912-929

12. Shapiro F, Glimcher MJ, Holtrop ME, Tashjian AH Jr, BrickleyParsons D, Kenzora JE (1980) Human osteopetrosis: a histological, ultrastructural, and biochemical study. J Bone Joint Surg Am 62(3): 384-399

13. Saluja S, Bhandari S, Aggarwal S, Kapoor S (2009) Osteopetrosis: a rare cause of anemia-review of literature. Indian J Pathol Microbiol 52(3):363-367. https://doi.org/10.4103/0377-4929. 54995

14. Sharma SS, Saravanan C, Sathyabama V, Satish C (2013) Osteopetrosis of the mandible masquerading as tubercular osteomyelitis. BMJ Case Rep. https://doi.org/10.1136/bcr-2012-007487

15. Sreehari S, Naik DR, Eapen M (2011) Osteopetrosis: a rare cause of anemia. Hematol Rep 3(1):e1. https://doi.org/10.4081/hr.2011.e1

16. Soliman DS (2017) Bone marrow biopsy findings in a case of rare infantile malignant osteopetrosis presented with bicytopenia and leukoerythroblastic picture. Human Pathol: Case Rep 10:10-11

17. Tolar J, Teitelbaum SL, Orchard PJ (2004) Osteopetrosis. N Engl J Med 351(27):2839-2849. https://doi.org/10.1056/NEJMra040952

18. Frattini A, Orchard PJ, Sobacchi C, Giliani S, Abinun M, Mattsson JP, Keeling DJ, Andersson AK, Wallbrandt P, Zecca L, Notarangelo LD, Vezzoni P, Villa A (2000) Defects in TCIRG1 subunit of the vacuolar proton pump are responsible for a subset of human autosomal recessive osteopetrosis. Nat Genet 25(3):343346. https://doi.org/10.1038/77131

19. Schulz AS, Moshous D, Steward CG, Villa A, Sobacchi C (2015) Osteopetrosis. Consensus guidelines for diagnosis, therapy and follow-up. Version 31108015

20. Wu CC, Econs MJ, DiMeglio LA, Insogna KL, Levine MA, Orchard PJ, Miller WP, Petryk A, Rush ET, Shoback DM, Ward LM, Polgreen LE (2017) Diagnosis and management of osteopetrosis: consensus guidelines from the osteopetrosis working group. J Clin Endocrinol Metab 102(9):3111-3123. https://doi.org/ 10.1210/jc.2017-01127

Publisher's note Springer Nature remains neutral with regard to jurisdictional claims in published maps and institutional affiliations. 